\title{
Momentum-dependent multiple gaps in magnesium diboride probed by electron tunnelling spectroscopy
}

\author{
Ke Chen ${ }^{1,2}$, Wenqing Dai ${ }^{1}$, C.G. Zhuang1,2, Qi Li ${ }^{1}$, Steve Carabello3 ${ }^{3}$ Joseph G. Lambert ${ }^{3}$, Jerome T. Mlack ${ }^{3}$, \\ Roberto C. Ramos ${ }^{3, \dagger} \&$ X. X. Xi $i^{1,2}$
}

The energy gap is the most fundamental property of a superconductor. $\mathrm{MgB}_{2}$, a superconductor discovered in 2001, exhibits two different superconducting gaps caused by the different electron-phonon interactions in two weakly interacting bands. Theoretical calculations predict that the gap values should also vary across the Fermi surface sheets of $\mathrm{MgB}_{2}$. However, until now, no such variation has been observed. It has been suggested that two gap values were sufficient to describe real $\mathrm{MgB}_{2}$ samples. Here we present an electron tunnelling spectroscopy study on $\mathrm{MgB}_{2}$ /native oxide/ $\mathrm{Pb}$ tunnel junctions that clearly shows a distribution of gap values, confirming the importance of the anisotropic electron-phonon interaction. The gap values, and their spreads found from the tunnel junction measurements, provide valuable experimental tests for various theoretical approaches to the multi-band superconductivity in $\mathrm{MgB}_{2}$.

\footnotetext{
${ }^{1}$ Department of Physics, The Pennsylvania State University, University Park, Pennsylvania 16802, USA. ${ }^{2}$ Department of Physics, Temple University, Philadelphia, Pennsylvania 19122, USA. ${ }^{3}$ Department of Physics, Drexel University, Philadelphia 19104, USA . †Present address: Division of Natural Sciences, Indiana Wesleyan University, Marion, Indiana 46953, USA. Correspondence and requests for materials should be addressed to K.C. (email: kchen@temple.edu).
} 
T here has been a surge of interest in multi-band superconductivity owing to its existence in $\mathrm{MgB}_{2}$ (refs 1-4), iron-based superconductors $^{5}$, and other superconducting materials ${ }^{6-8}$. The crystal structure of $\mathrm{MgB}_{2}$ is formed by layers of honeycombed boron atoms intercalated with magnesium atoms located above the centre of the boron hexagons. The boron $s, p_{x}$, and $p_{y}$ orbitals form quasi-twodimensional $\sigma$ bands, which couple strongly to the in-plane boron $E_{2 g}$ phonon mode and results in the formation of superconducting $\sigma$ gaps of about $7 \mathrm{meV}$. The boron $p_{z}$ orbit forms the three-dimensional $\pi$ bands that have a much weaker coupling to the $E_{2 g}$ mode, leading to $\pi$ gaps of about $2 \mathrm{meV}$. There is a finite coupling between the $\pi$ and $\sigma$ bands, and both gaps vanish at a common $T_{\mathrm{c}}$. Besides the two widely observed gaps ${ }^{9-12}$, first-principles calculations taking into account the fully anisotropic electron-phonon interaction predict that there should also be an intraband anisotropy within the $\sigma$ and $\pi$ gaps $^{1,2}$. In an earlier work Choi et al. solved the Eliashberg equation in the clean limit, using an anisotropic electron-phonon interaction $\lambda\left(\mathbf{k}, \mathbf{k}^{\prime}\right)$, and found an energy gap $\Delta(\mathbf{k})$ that spreads between 1.2 and $3.7 \mathrm{meV}$ for the $\pi$ bands and from 6.4 to $7.2 \mathrm{meV}$ for the $\sigma$ bands ${ }^{1,13}$. Later, direct first-principles calculations by Floris et al., using superconducting density functional theory without any semi-phenomenological parameters for the Coulomb repulsion, confirmed the existence of the anisotropy ${ }^{2}$. However, experimental evidence for a distribution of gap values, beyond just two values, has been lacking. Mazin et al. have suggested that the gap distribution cannot be resolved owing to the effects of intraband scattering ${ }^{14}$.

Electron tunnelling spectroscopy probes the quasiparticle density of states (DOS) in a superconductor, and thus provides the most direct measurement of the superconducting energy gap ${ }^{15}$. Previously, we have reported $\mathrm{MgB}_{2}$ /native oxide/ $\mathrm{Pb}$ tunnel junctions with bottom electrode being $c$-axis-oriented $\mathrm{MgB}_{2}$ film grown by hybrid physical-chemical vapour deposition (HPCVD) on (0001) single crystal $6 \mathrm{H}-\mathrm{SiC}$ substrate ${ }^{16}$. The predominant structure in the tunnelling spectra of these junctions is from the $\pi$ gap as the $\sigma$ carriers are mostly confined to the $a-b$ planes. When the $a-b$ planes of the bottom $\mathrm{MgB}_{2}$ electrode are exposed for electron tunnelling, such as in $\mathrm{MgB}_{2}$ films on $\mathrm{MgO}$ (211) substrate, where the $c$ axis is tilted by $19.5^{\circ}$ away from the film normal ${ }^{17}$, contributions from the $\sigma$ gap can also be observed. These tunnel junctions enable us to study both the $\pi$ and $\sigma$ gaps of $\mathrm{MgB}_{2}$.

In this study, we performed electron tunnelling spectroscopy measurements using $\mathrm{MgB}_{2} /$ native oxide/ $\mathrm{Pb}$ tunnel junctions made from HPCVD-grown $\mathrm{MgB}_{2}$ films on $\mathrm{MgO}$ (211), $\mathrm{SiC}$ (0001), and $c$-axis- $8^{\circ}$-tilted $\mathrm{SiC}$ substrates. The observed conductance peaks due to the $\sigma$ and $\pi$ gaps show fine structure, from which we derived the distribution of gap values within the two gaps. Most likely, the cleanliness of the $\mathrm{MgB}_{2}$ electrode and the high-quality $\mathrm{MgB}_{2} /$ oxide interface made the observation possible, because increased scattering in the $\mathrm{MgB}_{2}$ electrode and a high junction resistance were found to smear out the fine structure. The experimentally obtained gap distribution is in agreement with the first-principles calculations.

\section{Results}

Tunnelling spectra. The tunnelling conductance $\mathrm{d} I / \mathrm{d} V$ versus voltage $V$ for a $\mathrm{MgB}_{2} /$ native oxide/ $\mathrm{Pb}$ junction on $\mathrm{MgO}$ (211) at $T=$ $1.8 \mathrm{~K}$ is shown in Figure 1. Two sets of conductance peaks are seen in the spectrum. The peaks around $\pm 3.5 \mathrm{mV}$ correspond to $\pm\left(\Delta_{\pi}+\Delta_{\mathrm{Pb}}\right)$, and the peaks around $\pm 8.5 \mathrm{mV}$ to $\pm\left(\Delta_{\sigma}+\Delta_{\mathrm{Pb}}\right)$, where $\Delta_{\pi}, \Delta_{\sigma}$, and $\Delta_{\mathrm{Pb}}$ denote the superconducting gaps in the $\mathrm{MgB}_{2} \pi$ band, $\mathrm{MgB}_{2} \sigma$ band, and $\mathrm{Pb}$, respectively. The spectrum can be fitted to the standard tunnelling equation, assuming that the total normalized DOS $N(\varepsilon)$ is a linear combination of the contributions from the $\pi$ and $\sigma$ bands $\left(N_{\pi}\right.$ and $N_{\sigma}$, respectively), that is, $N(\varepsilon)=r N_{\pi}(\varepsilon)+(1-r) N_{\sigma}(\varepsilon)$. The coefficient $r$ represents the contribution of the $\pi$ band to the DOS, which is $89 \%$ for this junction ${ }^{17}$. It can be seen that both the $\pi$ and $\sigma$ peaks are not simple, smooth ones, but exhibit fine structure.

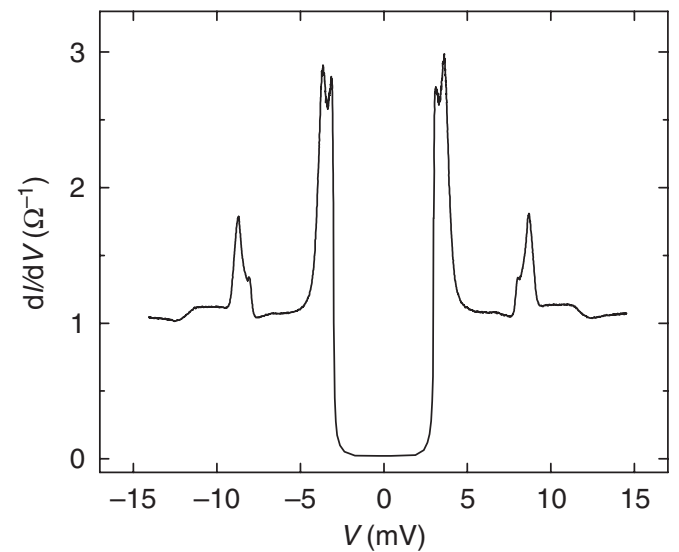

Figure 1 | Tunnelling spectrum of a $\mathbf{M g B}_{\mathbf{2}}$ /native oxide/ $\mathbf{P b}$ junction at $1.8 \mathrm{~K}$. Both the $\pi$-gap feature and the $\sigma$-gap feature show subtle peak structure.
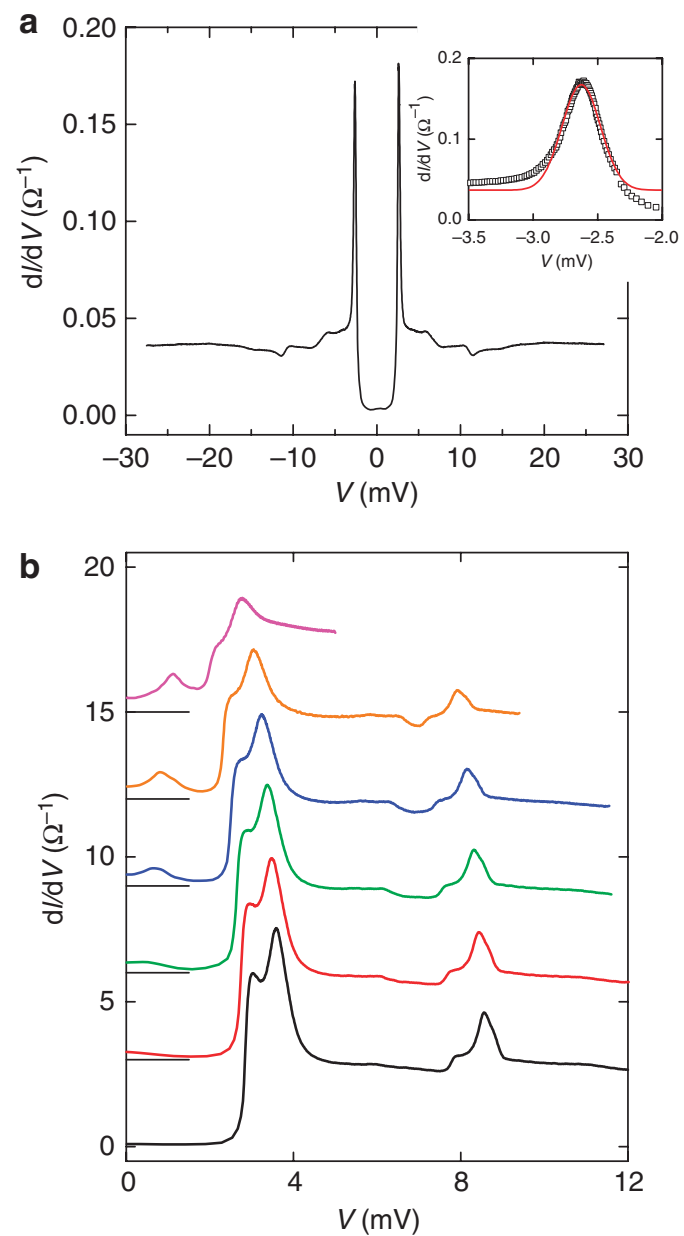

Figure $\mathbf{2}$ | The subtle gap peak structure is not caused by $\mathbf{P b}$.

(a) Tunnelling spectrum of a $\mathrm{Pb} / \mathrm{PbO} / \mathrm{Pb}$ junction at $T=4.2 \mathrm{~K}$. (inset) The single peak at about $-2.6 \mathrm{mV}$ corresponds to twice the average superconducting gap of $\mathrm{Pb}$. The experimental data (squares) are fit by a Gaussian function (line). (b) The tunnelling spectra of a $\mathrm{MgB}_{2}$ /native oxide/Pb junction at $T=4.2,5.0,5.5,6.0,6.5$ and $7.0 \mathrm{~K}$ (curves from bottom to top). The curves are shifted vertically for clarity with horizontal bars indicating zero conductance. 

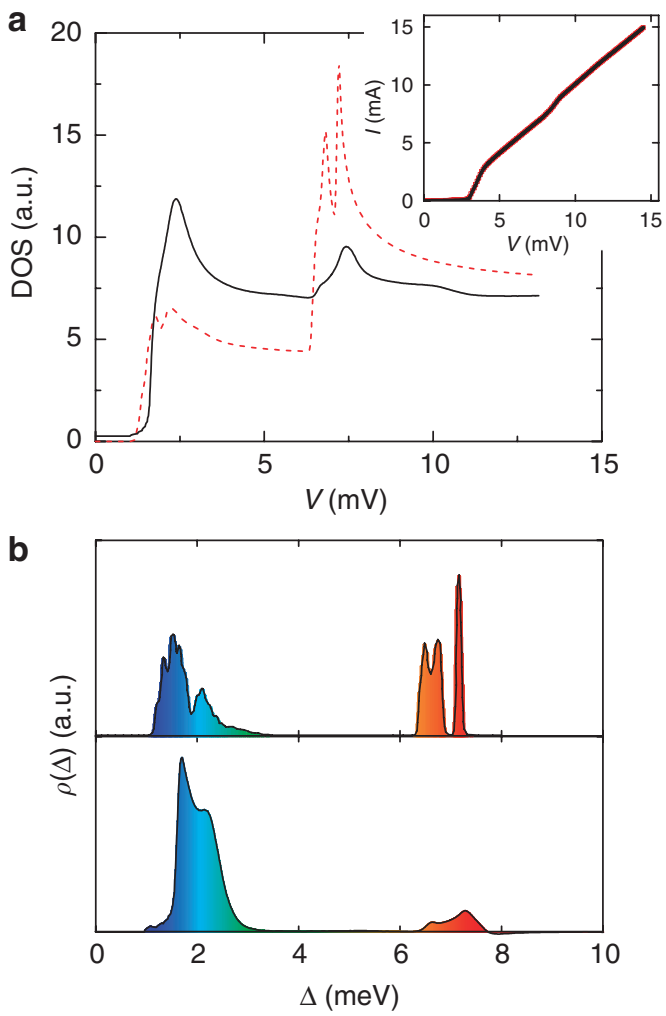

Figure 3 | The density of states and energy gap distribution of $\mathbf{M g B}_{\mathbf{2}}$. (a) The superconducting DOS of $\mathrm{MgB}_{2}$ at $T=0$ as derived from the tunnelling $I-V$ curve (solid line) and calculated (dashed line) from ref. 1 (inset). The tunnelling $I-V$ curve at $T=1.8 \mathrm{~K}$ as measured (black line) and reconstructed (red squares) from the derived distribution of $\mathrm{MgB}_{2}$ energygap values. (b) The distribution of $\mathrm{MgB}_{2}$ energy-gap values at $T=1.8 \mathrm{~K}$ derived from the superconducting DOS (bottom) compared with the theoretical results (top) of ref. 1 .

Subtle gap features are not caused by $\mathrm{Pb}$. To rule out the contribution of the $\mathrm{Pb}$ energy gap to the fine structure, we made $\mathrm{Pb} / \mathrm{PbO} / \mathrm{Pb}$ junctions with $\mathrm{Pb}$ films grown under the same conditions as the $\mathrm{MgB}_{2} /$ native oxide/ $\mathrm{Pb}$ junctions. A typical tunnelling spectrum is shown in Figure 2a. The sharp peaks at $V= \pm 2.62 \mathrm{mV}$ correspond to the double gap. The Gaussian fit in the inset to Figure 2 a shows only one single peak with full-width-at-half-maximum (FWHM) around $0.29 \mathrm{mV}$, indicating an average energy gap of about $1.31 \pm 0.01 \mathrm{meV}$ at $4.2 \mathrm{~K}$. This also suggests that the resolution of our tunnelling measurement at $4.2 \mathrm{~K}$ is better than $0.29 \mathrm{mV}$. Figure $2 \mathrm{~b}$ shows the tunnelling spectra of another $\mathrm{MgB}_{2} /$ native oxide/ $\mathrm{Pb}$ junction on $\mathrm{MgO}(211)$ at temperatures in the range of 4.2 to $7.0 \mathrm{~K}$, over which the gap of $\mathrm{Pb}$ changes substantially. Although the conductance peaks shift to lower voltages when the temperature increases, owing to the temperature dependence of the $\mathrm{Pb}$ gap, the basic fine structure does not change. Thus the fine structure can be definitively attributed to $\mathrm{MgB}_{2}$, as the $\mathrm{MgB}_{2}$ gaps are insensitive to temperature in this range $\left(T<T_{\mathrm{c}} / 2\right)$.

Deconvolution of tunnelling spectra. Because the normalized quasiparticle $\mathrm{DOS}$ of $\mathrm{Pb}$ is given by the BCS expression

$$
N_{\mathrm{Pb}}(E)=\operatorname{Re}\left(\frac{|E|}{\sqrt{E^{2}-\Delta_{\mathrm{Pb}}^{2}}}\right),
$$

where $\Delta_{\mathrm{Pb}}$ is $1.35 \mathrm{meV}$ at $1.8 \mathrm{~K}$, we can derive the DOS of $\mathrm{MgB}_{2}$ by deconvoluting the tunnelling $I-V$ curve using an approach first used by Blamire ${ }^{18}$. We do not consider the phonon structure of $\mathrm{Pb}$ at around $7 \mathrm{meV}$ and $11 \mathrm{meV}$ caused by the strong electron-phonon couplings ${ }^{19}$, because their effects are negligible compared with the contributions owing to the energy gaps. The tunnelling current of a $\mathrm{MgB}_{2} /$ native oxide/ $\mathrm{Pb}$ junction can be expressed as

$$
\begin{aligned}
I(V)= & \frac{1}{R_{n}} \int_{-\infty}^{\infty} N_{\mathrm{MgB}_{2}}(e u) \operatorname{Re}\left[\frac{|u+V|}{\sqrt{(u+V)^{2}-\left(\Delta_{\mathrm{Pb}} / e\right)^{2}}}\right] \\
& \times[f(e u)-f(e u+e V)] \mathrm{d} u,
\end{aligned}
$$

where $N_{\mathrm{MgB}_{2}}$ is the $\mathrm{MgB}_{2}$ DOS, $e$ is the electron charge, and $f$ is the Fermi distribution function ${ }^{15}$. At low temperatures, the region $\Delta_{\mathrm{m}} / e<u<V-\Delta_{\mathrm{Pb}} / e$ dominates the integration (greater than $99 \%$ for $V>2.8 \mathrm{mV}$ at $T=1.8 \mathrm{~K}$ ), where $\Delta_{\mathrm{m}} \sim 1.3 \mathrm{meV}$ is the smallest gap value for $\mathrm{MgB}_{2}$. The points of the $\mathrm{MgB}_{2}$ DOS are sequentially obtained from the tunnelling spectrum shown in Figure 1 by discretizing the integrations over successively larger ranges ${ }^{18}$. The $\mathrm{MgB}_{2} \mathrm{DOS}$ is shown in Figure 3a, together with the DOS, calculated by Choi et al. ${ }^{1}$ The gap distribution $\rho(\Delta)$ is related to the DOS by the relation

$$
N_{\mathrm{MgB}_{2}}(E)=\int_{0}^{E} \frac{E}{\sqrt{E^{2}-\Delta^{2}}} \rho(\Delta) \mathrm{d} \Delta
$$

The gap distribution found by deconvoluting the DOS is shown in Figure $3 \mathrm{~b}$. The validity of the deconvolution algorithms is justified by the overlap between the experimental $I-V$ curve and the reconstructed $I-V$ curve from the derived $\rho(\Delta)$, as shown in the inset of Figure 3a.

Effects of electron scattering on gap features. In Figure 4a, the gap distribution normalized to the peak value of $\rho(\Delta)$ is shown for several other $\mathrm{MgB}_{2} /$ native oxide/ $\mathrm{Pb}$ junctions on different substrates. The $R_{n} A$ product, where $R_{n}$ is the junction normal-state resistance and $A$ is the junction area, ranges from 0.82 to $7.7 \mathrm{~m} \Omega \mathrm{cm}^{2}$. As the intraband scattering rate in the $\mathrm{MgB}_{2}$ electrode is very sensitive to details of the deposition conditions, it varies from junction to junc$\operatorname{tion}^{20}$. As a result, the fine structures in the gap distributions are more distinct in some junctions than in others. Because of epitaxial tensile strain ${ }^{21}, \mathrm{MgB}_{2}$ films on $\mathrm{SiC}$ substrates have higher $T_{c} \mathrm{~s}$ (over $41 \mathrm{~K}$ ) than films on $\mathrm{MgO}$ substrates (about $38.3 \mathrm{~K}$ ), and consequently the average $\sigma$ gap is larger. To vary the scattering rate systematically, $\mathrm{N}_{2}$ gas (99.999\% purity) was added to the carrier gas during the $\mathrm{MgB}_{2}$ deposition, since the trace amount of oxygen in $\mathrm{N}_{2}$ leads to scattering centres in the $\mathrm{MgB}_{2}$ films ${ }^{22}$. Figure $4 \mathrm{~b}$ shows the $\pi$ peaks in $\rho(\Delta)$ for four junctions on $\mathrm{SiC}(0001)$ substrates with different flow rates of $\mathrm{N}_{2}$ addition. As seen in the inset, the electron mean free path, derived from the electrical resistivity, becomes shorter and the energy distribution peak of the $\pi$ gap becomes sharper (smaller FWHM) with increasing amounts of $\mathrm{N}_{2}$ addition. This indicates that the intraband scattering smears out the gap distribution. Collectively, the results shown in Figure 4a,b indicate that the observed gap values and their distributions are sensitively affected by the electronic scattering in the $\mathrm{MgB}_{2}$ films.

\section{Discussion}

The gap distribution derived from the tunnelling spectrum is in agreement with the first-principles calculations ${ }^{1,2}$. Both the $\pi$ and $\sigma$ gaps split into several peaks with the peak positions similar to those predicted by Choi et al. ${ }^{1}$ as can be seen from Figure $3 \mathrm{~b}$. The spread in values for the $\pi$ gap is $2.3 \mathrm{meV}$ and is $1.3 \mathrm{meV}$ for the $\sigma$ gap, which are close to the spreads predicted by Choi et al.. The results are also compatible with preliminary superconducting density functional theory calculations by Massidda, Sanna, and Profeta (personal communication) performed with a full $k$-space resolution. As different groups are using different theoretical approaches to $\mathrm{MgB}_{2}$ and have produced differing values of the electron-phonon coupling coefficients, our results can be used to facilitate experimental verification 

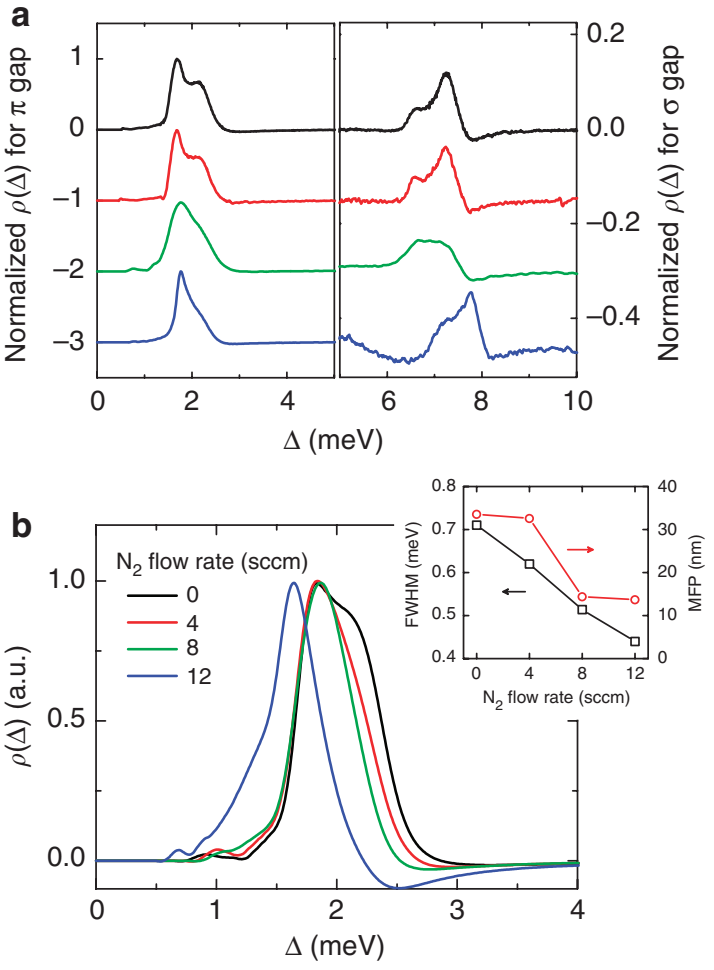

Figure 4 | Variation in the energy gap distribution in different $\mathbf{M g B}_{\mathbf{2}}$ junctions. (a) The energy gap distributions for four junctions: A (black), B (red), C (Green) on MgO (211) and D (blue) on c-axis-8 ${ }^{\circ}$-tilted SiC. Each curve is normalized to its peak value and shifted vertically for clarity. Curves for junctions $\mathrm{A}$ and $\mathrm{C}$ were obtained at $T=1.8 \mathrm{~K}$ and those for junctions $B$ and $D$ were obtained at $T=4.2 \mathrm{~K}$. The values of the product $R_{n} A$ are $1.6,0.82,7.7$ and $2.6 \mathrm{~m} \Omega \mathrm{cm}^{2}$ for junctions $A, B, C$ and $D$, respectively. The $\sigma$-gap curve for junction $D$ is magnified five times. (b) The energy gap distribution around $\pi$ gap region for 4 junctions on $\mathrm{SiC}(0001)$ substrates at $T=4.2 \mathrm{~K}$ with different $\mathrm{N}_{2}$ flow rates during the $\mathrm{MgB}_{2}$ growth. (inset) The dependence of FWHM of the gap distribution peaks and the electron mean free path (MFP) to the flow rate of $\mathrm{N}_{2}$ addition.

of theories. The magnitudes of peaks for $6 \mathrm{meV}<\Delta_{\sigma}<8 \mathrm{meV}$ are much smaller than for the range $1 \mathrm{meV}<\Delta_{\pi}<3 \mathrm{meV}$, which is opposite to the theoretical predictions. (Similar discrepancies are seen in the DOS shown in Fig. 3a.) The most probable reason is that, in our junctions, the $\sigma$-band charge carriers account for only $11 \%$ of the total tunnel current, whereas the theoretical result is effectively for isotropic tunnelling, where the $\sigma$-band contribution is around $33 \%$ (ref. 23).

Mazin et al. have pointed out that the fine structure in the distribution of gaps cannot be experimentally observed unless the intraband scattering rate satisfies $\gamma\langle\sqrt{\langle\Delta\rangle \delta \Delta}$, where $\langle\Delta\rangle$ is the average gap value for the band and $\delta \Delta$ is the variation of the gap value for the band ${ }^{14}$. They estimated that the electron mean free path of the $\mathrm{MgB}_{2}$ sample has to exceed $150 \mathrm{~nm}$. Taking the values of $\langle\Delta\rangle$ and $\delta \Delta$ from Figure $3 \mathrm{~b}$, the maximum intraband scattering rates in the $\mathrm{MgB}_{2}$ film are estimated to be 0.9 and $1.7 \mathrm{meV}$ for the $\pi$ and $\sigma$ bands, respectively. In the clean $\mathrm{MgB}_{2}$ film grown by HPCVD, the mean free path can be as large as $280 \mathrm{~nm}$ (ref. 24), which is most likely the reason that the fine structure in the gap distributions becomes observable in our junctions. The sensitivity of the fine structure in the gap distribution to the scattering rate shown in Figure $4 \mathrm{a}$ and the smearing out of the structure by the increasing number of scattering centres shown in Figure $4 \mathrm{~b}$ are all in agreement with the multiband theory of Mazin et al. ${ }^{14}$
Another factor that can affect the fine structure in the gap distribution is the scattering at the superconductor/insulator interfaces, which may cause all the states in the $\sigma$ or $\pi$ bands to mix. Indeed, the fine structure is smeared out in junctions with large values of the product $R_{n} A$, which, besides being due to thicker barriers, can also be partially due to strong interface scattering. It is not clear whether further fine structure will be observed if the scattering in $\mathrm{MgB}_{2}$ is reduced, or whether the observed resolution in the gap distribution already represents the limit imposed by surface mixing of carriers in the bands with different momenta.

In summary, we have studied tunnelling spectroscopy in $\mathrm{MgB}_{2} /$ native oxide/ $\mathrm{Pb}$ tunnel junctions and have found a distribution of the superconducting gap values for the $\sigma$ and $\pi$ bands in $\mathrm{MgB}_{2}$. This is possible because the $\mathrm{MgB}_{2}$ electrode grown by HPCVD was clean and had a long mean free path. In dirty single-band superconductors, the energy gap is single-valued because the scattering smears out the momentum dependence of the electron-phonon interaction ${ }^{25}$. For dirty $\mathrm{MgB}_{2}$ samples, both the $\sigma$ and $\pi$ gaps are single-valued owing to the effect of intraband scattering, but they remain distinct from each other because the interband scattering rate is small owing to the disparity of electronic structure of the two bands ${ }^{26}$. When the $\mathrm{MgB}_{2}$ sample is clean, the distribution of the energy-gap values becomes important. One dramatic consequence of the gap distribution is the smearing of the DOS singularity at the gap (Fig. 3a) and the absence of a sharp rise in current in the $I-V$ curves of tunnel junctions (inset of Fig. 3a). These are properties of clean superconductor junctions that must be confronted when considering their applications ${ }^{27}$.

\section{Methods}

Fabrication of tunnel junctions. To fabricate the $\mathrm{MgB}_{2}$ /native oxide/ $\mathrm{Pb}$ junctions, a $100-\mathrm{nm}$ thick $\mathrm{MgB}_{2}$ bottom electrode layer was first epitaxially grown on a $\mathrm{MgO}$ (211), $\mathrm{SiC}(0001)$, or $c$-axis- $8^{\circ}$-tilted $\mathrm{SiC}$ substrate by HPCVD. Because of the high purity of the $\mathrm{Mg}$ and $\mathrm{B}$ sources and the reducing hydrogen atmosphere during the deposition, $\mathrm{MgB}_{2}$ films, prepared by HPCVD, are exceptionally clean 24,28 . The mean free path of the $\mathrm{MgB}_{2}$ films, as estimated by the residue resistivity, can be greater than $280 \mathrm{~nm}$ and, in our case, is limited by the thickness of the film $(\sim 100 \mathrm{~nm})^{29}$. The transition temperature $T_{c}$ of the $\mathrm{MgB}_{2}$ films on $\mathrm{MgO}$ substrates is around $38.3 \mathrm{~K}$ and is $41.2 \mathrm{~K}$ on SiC substrates. To vary the scattering rate in $\mathrm{MgB}_{2}$ films, $\mathrm{N}_{2}$ gas (99.999\% purity) with flow rate from 0 to $12 \mathrm{sccm}$ (standard cubic centimetres per minute) was introduced along with $1,200 \mathrm{sccm} \mathrm{H}_{2}$ and $20 \mathrm{sccm}$ $\mathrm{B}_{2} \mathrm{H}_{6}\left(5 \%\right.$ in $\left.\mathrm{H}_{2}\right)$ during the $\mathrm{MgB}_{2}$ growth. The $\mathrm{MgB}_{2}$ film was then exposed to air at room temperature to form a native oxide barrier ${ }^{30}$. The bottom electrode stripe was subsequently created by painting the sample with Duco Cement. The 200$\mathrm{nm}$-thick $\mathrm{Pb}$ top electrode stripes, orthogonal to the bottom electrode stripe, were formed by thermal evaporation through a shadow mask at a background vacuum of around $1 \times 10^{-5} \mathrm{mbar}$. The junction size was about $0.3 \mathrm{~mm} \times 0.3 \mathrm{~mm}$.

Tunnelling spectra measurements. Four-probe electrical measurements were carried out in a Physical property measurement system at temperatures down to $1.8 \mathrm{~K}$. During the measurement, an in-plane magnetic field up to $1 \mathrm{mT}$ was applied to suppress the Josephson current. The tunnelling $I-V$ curves were acquired in current-bias mode and the tunnelling conductance $\mathrm{d} I / \mathrm{d} V-V$ curves were acquired by a lock-in amplifier with a $262 \mathrm{~Hz} 50 \mu \mathrm{V}$ root-mean-square modulation applied to the sample. The integration time constant was $1 \mathrm{~s}$ with a bias sweep rate outside the $\pi$ gap of about $20 \mu \mathrm{V} \mathrm{s}^{-1}$.

\section{References}

1. Choi, H. J., Roundy, D., Sun, H., Cohen, M. L. \& Louie, S. G. The origin of the anomalous superconducting properties of $\mathrm{MgB}_{2}$. Nature 418, 758-760 (2002).

2. Floris, A. et al. Superconducting properties of $\mathrm{MgB}_{2}$ from first principles. Physica C 456, 45-53 (2007).

3. Xi, X. X. Two-band superconductor magnesium diboride. Rep. Prog. Phys. 71, 116501 (2008).

4. Mazin, I. I. \& Antropov, V. P. Electronic structure, electron-phonon coupling, and multiband effects in $\mathrm{MgB}_{2}$. Physica C 385, 49-65 (2003).

5. Paglione, J. \& Greene, R. L. High-temperature superconductivity in iron-based materials. Nat. Phys. 6, 645-658 (2010).

6. Yokoya, T. et al. Fermi Surface Sheet Dependent Superconductivity in $2 \mathrm{H}$ $\mathrm{NbSe}_{2}$. Science 294, 2518-2520 (2001).

7. Jourdan, M., Zakharov, A., Foerster, M. \& Adrian, H. Evidence for multiband superconductivity in the heavy fermion compound $\mathrm{UNi}_{2} \mathrm{Al}_{3}$. Phys. Rev. Lett. 93, 097001 (2004). 
8. Nakajima, Y., Nakagawa, T., Tamegai, T. \& Harima, H. Specific-heat evidence for two-gap superconductivity in the ternary-iron silicide $\mathrm{Lu}_{2} \mathrm{Fe}_{3} \mathrm{Si}_{5}$. Phys. Rev. Lett. 100, 157001 (2008).

9. Iavarone, M. et al. Two-band superconductivity in $\mathrm{MgB}_{2}$. Phys. Rev. Lett. 89, 187002 (2002).

10. Bouquet, F., Fisher, R. A., Phillips, N. E., Hinks, D. G. \& Jorgensen, J. D. Specific heat of $\mathrm{Mg}^{11} \mathrm{~B}_{2}$ : Evidence for a second energy gap. Phys. Rev. Lett. 87, 047001 (2001).

11. Uchiyama, H. et al. Electronic structure of $\mathrm{MgB}_{2}$ from angle-resolved photoemission spectroscopy. Phys. Rev. Lett. 88, 157002 (2002).

12. Schmidt, H., Zasadzinski, J. F., Gray, K. E. \& Hinks, D. G. Evidence for twoband superconductivity from break-junction tunneling on $\mathrm{MgB}_{2}$. Phys. Rev. Lett. 88, 127002 (2002).

13. Choi, H. J., Roundy, D., Sun, H., Cohen, M. L. \& Louie, S. G. First-principles calculation of the superconducting transition in $\mathrm{MgB}_{2}$ within the anisotropic Eliashberg formalism. Phys. Rev. B 66, 020513 (2002).

14. Mazin, I. I. et al. Comment on 'First-principles calculation of the superconducting transition in $\mathrm{MgB}_{2}$ within the anisotropic Eliashberg formalism. Phys. Rev. B 69, 056501 (2004).

15. Wolf, E. Principles of Electron Tunneling Spectroscopy (Oxford University Press, USA, 1985).

16. Cui, Y., Chen, K., Li, Q., Xi, X. X. \& Rowell, J. M. Degradation-free interfaces in $\mathrm{MgB}_{2}$ /insulator/Pb Josephson tunnel junctions. Appl. Phys. Lett. 89, 202513 (2006).

17. Chen, K. et al. Study of $\mathrm{MgB}_{2} / \mathrm{I} / \mathrm{Pb}$ tunnel junctions on $\mathrm{MgO}$ (211) substrates. Appl. Phys. Lett. 93, 012502 (2008)

18. Blamire, M. G. Deconvolution of tunneling density of states from superconductor-insulator-superconductor current versus voltage data. Physica C 211, 467-474 (1993).

19. McMillan, W. L. \& Rowell, J. M. in Superconductivity (ed. R. D. Parks) Vol 1, p. 561 (Dekker, 1969).

20. Yang, H. et al. Fully Band-Resolved Scattering Rate in $\mathrm{MgB}_{2}$ Revealed by the Nonlinear Hall Effect and Magnetoresistance Measurements. Phys. Rev. Lett. 101, 067001 (2008).

21. Pogrebnyakov, A. V. et al. Enhancement of the superconducting transition temperature of $\mathrm{MgB}_{2}$ by a strain-induced bond-stretching mode softening. Phys. Rev. Lett. 93, 147006 (2004).

22. Cui, Y. Magnesium Diboride Thin Films and Devices. PhD thesis (Pennsylvania State University USA, 2007).

23. Brinkman, A. et al. Multiband model for tunneling in $\mathrm{MgB}_{2}$ junctions. Phys. Rev. B 65, 180517(R) (2002).

24. Xi, X.X. et al. $\mathrm{MgB}_{2}$ thin films by hybrid physical-chemical vapor deposition. Physica C 456, 22-37 (2007).
25. Anderson, P. W. Theory of dirty superconductors. J. Phys. Chem. Solids 11, 26-30 (1959).

26. Mazin, I. I. et al. Superconductivity in $\mathrm{MgB}_{2}$ : clean or dirty? Phys. Rev. Lett. 89, 107002 (2002).

27. Chen, K. et al. High- $\mathrm{J}_{\mathrm{c}} \mathrm{MgB}_{2}$ Josephson junctions with operating temperature up to 40 K. Appl. Phys. Lett. 96, 042506 (2010).

28. Zeng, X. H. et al. In situ epitaxial $\mathrm{MgB}_{2}$ thin films for superconducting electronics. Nat. Mater. 1, 25-38 (2002).

29. Pogrebnyakov, A.V. et al. Thickness dependence of the properties of epitaxial $\mathrm{MgB}_{2}$ thin films grown by hybrid physical-chemical vapour deposition. Appl. Phys. Lett. 82, 4319-4321 (2003).

30. Cui, Y., Chen, K., Li, Q., Xi, X. X. \& Rowell, J. M. MgB $/$ /insulator/Pb Josephson junctions with different tunnel barriers. IEEE Trans. Appl. Supercond. 17, 218-221 (2007).

\section{Acknowledgements}

We thank John M. Rowell, Marvin L. Cohen, Hyoung J. Choi, Steven G. Louie, Igor I. Mazin and Sandro Massidda for helpful discussions and unpublished data. We are greatful to Peter Riseborough for editing the manuscript. The work is partially funded by ONR under Grant No. N00014-07-1-0079 (X.X.X.), and by DOE under Grant No. DEFG02-08ER46531 (Q.L.). This study was supported by the Pennsylvania State University Materials Research Institute Nanofabrication Lab and the National Science Foundation Cooperative Agreement No. ECS-0335765.

\section{Author contributions}

K.C. designed and carried out the experiment, analysed the data and did the calculations. K.C. and X.X.X. wrote the manuscript. W.D. and C.G.Z. took part in sample fabrication, data analysis and measurements. S.C., J.G.L., J.T.M. and R.C.R. carried out an independent junction measurement at $\mathrm{mK}$ temperatures. X.X.X., Q.L. and R.C.R. were responsible for the research activities that generated the data and the understanding of the results.

\section{Additional information}

Competing financial interests: The authors declare no competing financial interests.

Reprints and permission information is available online at http://npg.nature.com/ reprintsandpermissions/

How to cite this article: Chen, K. et al. Momentum-dependent multiple gaps in magnesium diboride probed by electron tunnelling spectroscopy. Nat. Commun. 3:619 doi: $10.1038 /$ ncomms 1626 (2012) 